\title{
Spikelet Sterility Studies on Induced Mutant Populations of Cluster Bean [Cyamopsis tetragonoloba (L.) Taub.]
}

\author{
N.M. Meghana ${ }^{1}$, R.K. Ramachandra ${ }^{2 *}$, B. Fakrudin ${ }^{1}$, H.M. Pallavi ${ }^{2}$, \\ Vishnuvardhana ${ }^{1}$, M. Anjanappa ${ }^{1}$ and A. Harish ${ }^{3}$ \\ ${ }^{1}$ College of Horticulture, Bengaluru, Karnataka, India \\ ${ }^{2}$ College of Horticulture, Mysore, Karnataka, India \\ ${ }^{3}$ College of Horticulture, VCSG UUHF, Bharsar, Uttarakhand, India \\ *Corresponding author
}

\begin{tabular}{|l|}
\hline Ke y w o r d s \\
$\begin{array}{l}\text { Gamma radiation, } \\
\text { Mutation, Cluster } \\
\text { bean, Sterility }\end{array}$ \\
\hline Article Info \\
\hline $\begin{array}{l}\text { Accepted: } \\
\text { 10 October } 2018 \\
\text { Available Online: } \\
10 \text { November } 2018\end{array}$ \\
\hline
\end{tabular}

\section{A B S T R A C T}

Mutation breeding is one of the best ways to induce genetic variability within a crop species in a short period of time. It is equally a potentially powerful tool for cluster bean as other crops for genetic enrichment. The gamma radiation induced $190 \mathrm{M}_{4}$ mutant lines of cluster bean [Cyamopsis tetragonoloba (L.) Taub.] obtained from Centre for Biotechnological Research (CBR), Dept. of BCI, College of Horticulture, Bengaluru and which were used for the field experiment on spikelet sterility during kharif 2017 (June November) at College of Horticulture, Mysore. The present investigation revealed the presence of the 12 sterile mutant lines viz., $\mathrm{A}_{5}, \mathrm{~A}_{16}, \mathrm{~A}_{23}, \mathrm{~B}_{52}, \mathrm{~B}_{60}, \mathrm{~B}_{61}, \mathrm{C}_{93}, \mathrm{C}_{94}, \mathrm{C}_{102}, \mathrm{D}_{124}$, $\mathrm{D}_{136}$ and $\mathrm{D}_{141}$. The sterile mutants morphologically differ from fertile mutants in flowering, pod setting and development vegetative growth. There was no or very less amount reproductive growth is noticed in these mutants with small rudimentary like pod growth and 1 or 2 pods are observed with 1 to 3 seeds per pod with very long vegetative growth. The sterility was confirmed through visual observations and the pollen fertility percentage was recorded by doing the acetocarmine smear test. The stained pollens are considered as fertile and non-stained pollens are sterile because the pollens are non-viable in case of sterile plants and the fertile mutants are observed with $82.02 \%\left(\mathrm{D}_{160}\right)$ to $99.72 \%\left(\mathrm{D}_{163}\right)$ per cent pollen fertility while, the pollen fertility was $5.06 \%\left(\mathrm{~B}_{60}\right)$ to $10.04 \%\left(\mathrm{D}_{141}\right)$ in case of sterile mutant plants.

\section{Introduction}

Cluster bean [Cyamopsis tetragonoloba (L.) Taub.] [2n=14], is a neglected arid legume for a long time and under exploited leguminous vegetable crop belonging to the family Fabaceae. It is commonly known as guar, chavli kayi, guari kayi and khutti. Cluster bean is native to the Indian subcontinent (Sanghi et al., 1964). India is the largest producer of cluster bean and contributes $75-82 \%$ of the total cluster bean production in the world. In South India it is being cultivated for vegetable purpose. The pods grown in clusters give the common name cluster bean. It is an important and potential vegetable cum industrial crop grown for its tender pods for vegetable purpose and also for endospermic gum, which ranges between 30-35 per cent (Tawar et al., 1988). 
Considering the importance as a vegetable and its adaptability to arid drought conditions, there is a prime need for its improvement. This calls for an evaluation of local or related genotypes to know the variability and mean performance. But it is a self-pollinated crop, which ensures the limited variability with in the crop and nothing can be achieved with this so the breeder will have to enrich the germplasm to create greater variability through hybridization, mutation and polyploidy breeding. Mutation breeding is one of the best ways to induce genetic variability within a crop species in a short period of time. It is a potentially powerful tool for cluster bean enrichment, since it has very limited exploitable and useful genetic variation. The creation of variability through hybridization is very difficult because of very small and delicate flower structures, which often result in very poor seeds setting in the manually hybridized buds and higher frequency of flower drop during and after crossing. Due to these reasons, not much enticing and fetching genetic variability has been generated through conventional breeding approaches in cluster bean. Looking at this limitation, efforts were initiated to create variability in cluster bean by using the tool of induced mutations. The present investigation was therefore undertaken to study the sterility related attributes among the $\mathrm{M}_{4}$ mutants of Cluster bean to identify the variability created in different traits by morphological observations.

\section{Materials and Methods}

The experiment was carried out at the PG research block, near National Horticultural Mission (NHM), College of Horticulture, Mysuru, during the year 2017- 18 involving the $194 \mathrm{M}_{3}$ mutant lines and three checks which were field evaluated in 8 blocks in an Augmented Block Design with repeated checks in each block. A set of $190 \mathrm{M}_{4}$ mutant line seeds of cluster bean obtained from
Centre for Biotechnology Research (CBR) Department of $\mathrm{BCI}, \mathrm{COH}$, Bengaluru were planted at a spacing of $45 \times 25 \mathrm{~cm}$ on $14^{\text {th }}$ of June 2017. The experiment was laid out following the recommended package of practices of UHS, Bagalkot for cluster bean (Anonymous 2016). The data was recorded on days to 50 per cent flowering number of days to 50 per cent maturity, number of days to harvest, plant height, number of branches per plant, pod breadth, pod length, number of pods per cluster, number of clusters per plant, number of pods per plant, ten pods weight $(\mathrm{g})$, pod yield per plant $(\mathrm{g})$, seeds per pod, seed yield per plant (g), 100-seed weight (g) and Acetocarmine test.

\section{Results and Discussion}

Variability present in the growth and sterility related characters was assessed through a simple approach of examining the range of variation. The study indicated presence of sufficient amount of variation among the mutants for all the characters studied. These results were in accordance with Dabas et al., (1982), Anila and Balakrishnan (1990), Hanchinamani (2004), Saini et al., (2010) and Girish et al., (2012), in all these studies different sets of cluster bean genotypes was field evaluated. The following are the sterile mutants observed in the present study viz $\mathrm{A}_{5}$, $\mathrm{A}_{16}, \mathrm{~A}_{23}$ (Plate 1), $\mathrm{B}_{52}, \mathrm{~B}_{60}, \mathrm{~B}_{61}$ (Plate 2), $\mathrm{C}_{93}$, $\mathrm{C}_{94}, \mathrm{C}_{102}$ (Plate 3), $\mathrm{D}_{124}, \mathrm{D}_{136}$ and $\mathrm{D}_{141}$ (Plate 4). These were studied to explore the variability for reproductive sterility related traits in selected $\mathrm{M}_{4}$ mutants of cluster bean.

The sterile mutant plants morphologically differ from fertile mutants $i$. e., only flowering and no pod setting and development is observed with the more vegetative growth and no or very less amount of reproductive growth is noticed in these mutants with small rudimentary like pod growth and 1 or 2 pods are observed with 1 to 3 seeds per pod. 
Table.1 Mean performance of sterile $\mathrm{M}_{4}$ mutants identified in cluster bean with their characteristic features

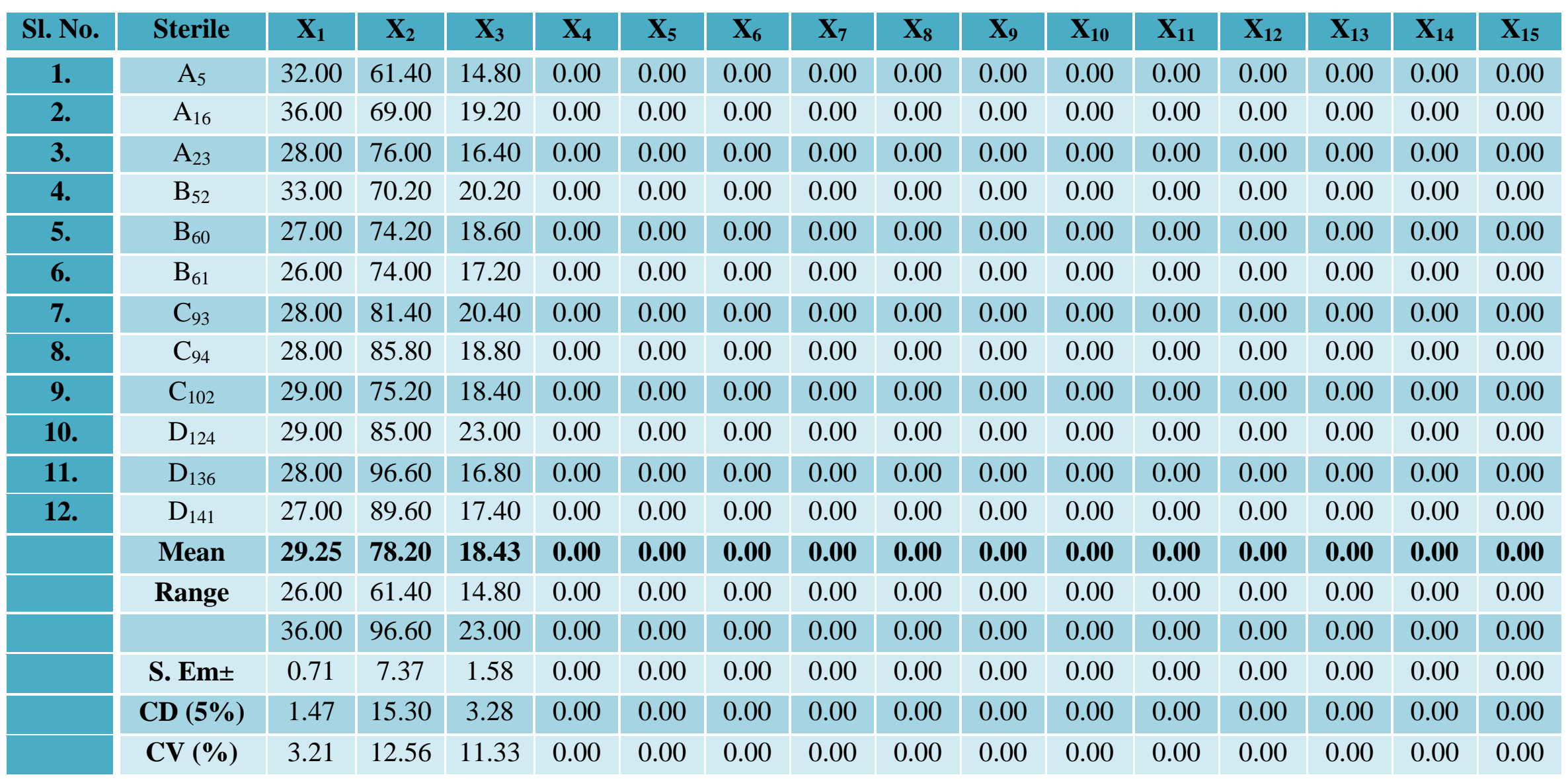

\begin{tabular}{|l|l|l|}
\hline $\mathbf{X}_{\mathbf{1}}=$ Days for $\mathbf{5 0 \%}$ flowering & $\mathbf{X}_{\mathbf{6}}=$ Pod width $(\mathbf{c m})$ & $\mathbf{X}_{11}=$ Ten pods weight $(\mathbf{g})$ \\
\hline $\mathbf{X}_{\mathbf{2}}=$ Plant height $(\mathbf{c m})$ & $\mathrm{X}_{7}=$ Days for harvest & $\mathrm{X}_{12}=$ Pod yield/plant $(\mathrm{g})$ \\
\hline $\mathbf{X}_{\mathbf{3}}=$ Branches/plant & $\mathrm{X}_{8}=$ Pods/cluster & $\mathrm{X}_{13}=$ Seeds/pod \\
\hline $\mathbf{X}_{\mathbf{4}}=$ Pod length $(\mathbf{c m})$ & $\mathrm{X}_{9}=$ Pod clusters/plant & $\mathrm{X}_{14}=$ Seed yield/plant $(\mathrm{g})$ \\
\hline $\mathbf{X}_{\mathbf{5}}=$ Days for 50\% maturity & $\mathrm{X}_{10}=$ Pods/plant & $\mathrm{X}_{15}=100$ seed weight $(\mathrm{g})$ \\
\hline
\end{tabular}




\section{Int.J.Curr.Microbiol.App.Sci (2018) 7(11): 1288-1298}

Plate.1 Sterile types observed in A-series of $\mathrm{M}_{4}$ mutants of cluster bean
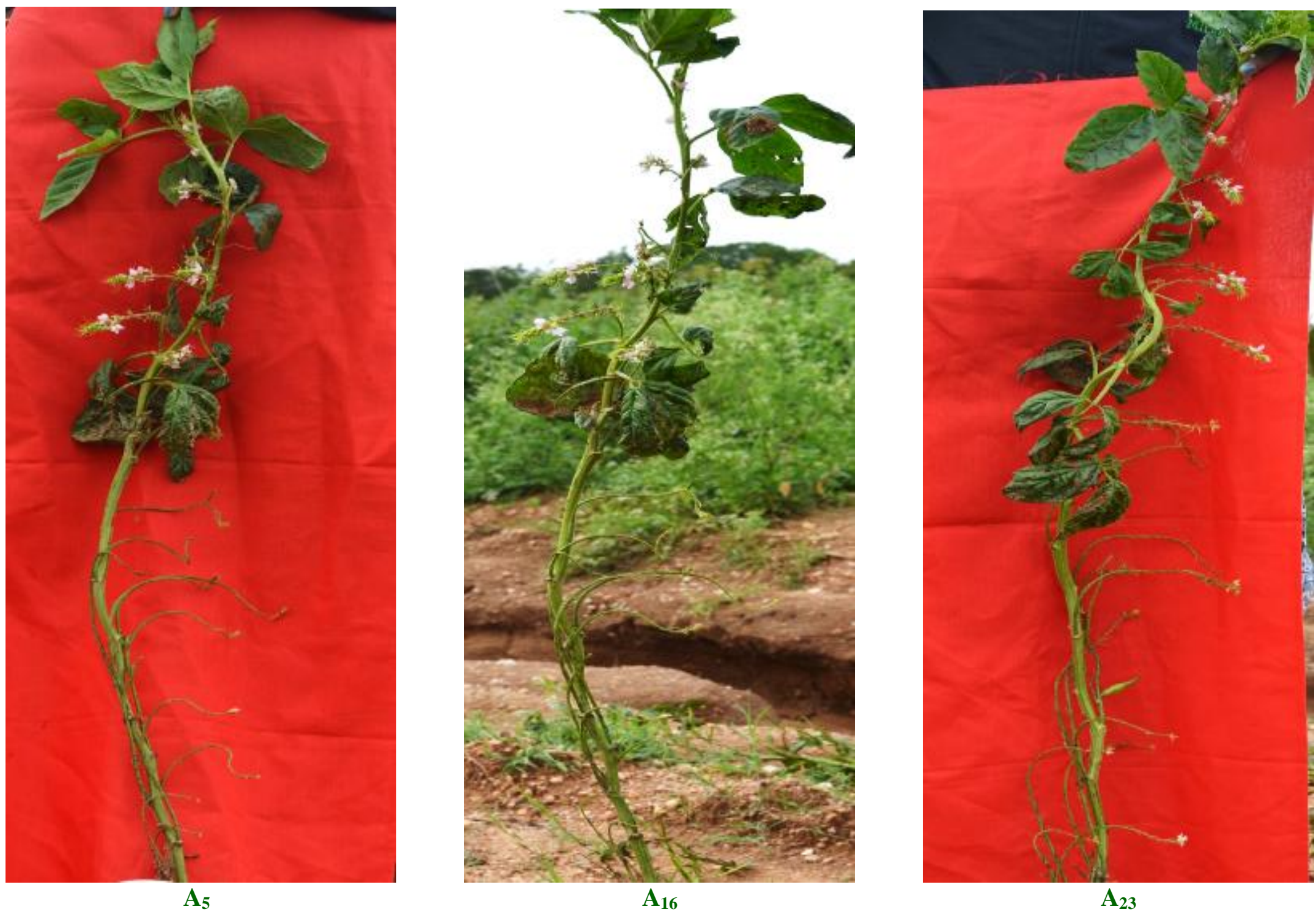


\section{Int.J.Curr.Microbiol.App.Sci (2018) 7(11): 1288-1298}

Plate.2 Sterile types observed in B-series of $\mathrm{M}_{4}$ mutants of cluster bean

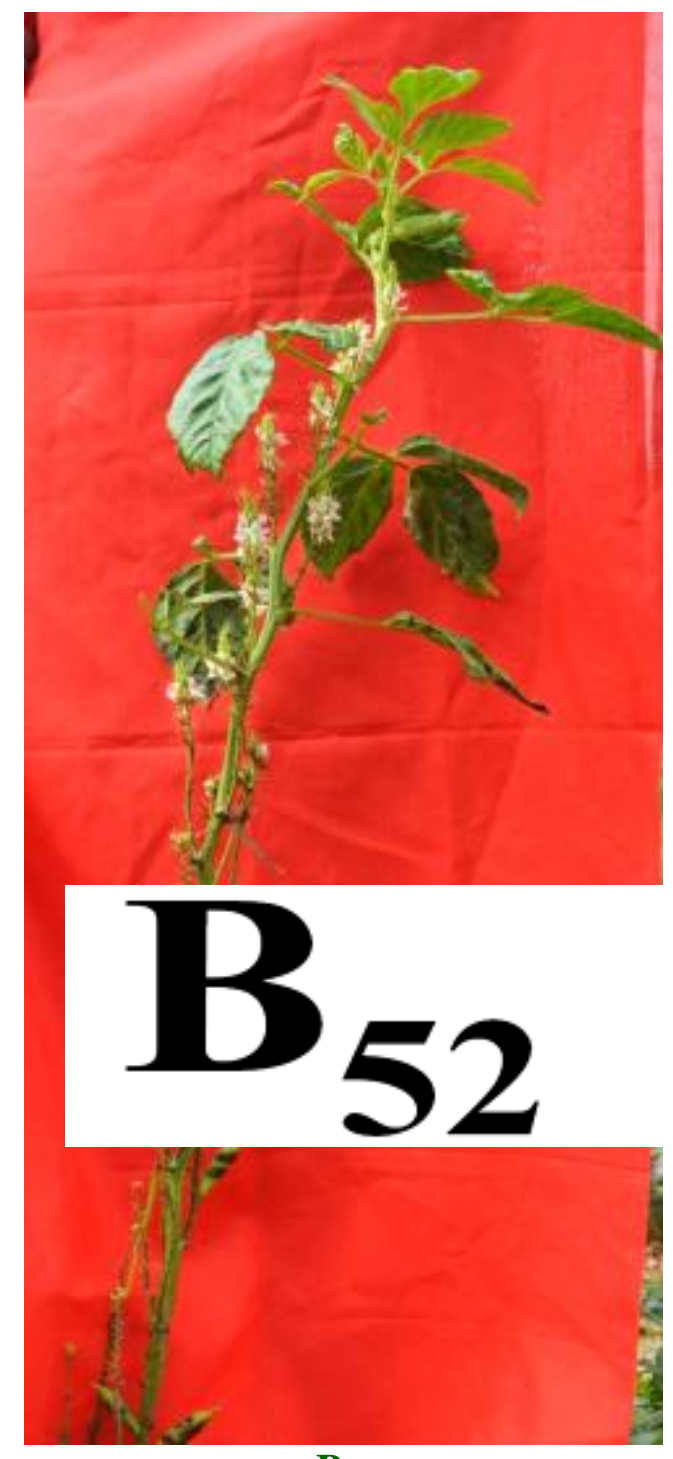

$\mathbf{B}_{52}$

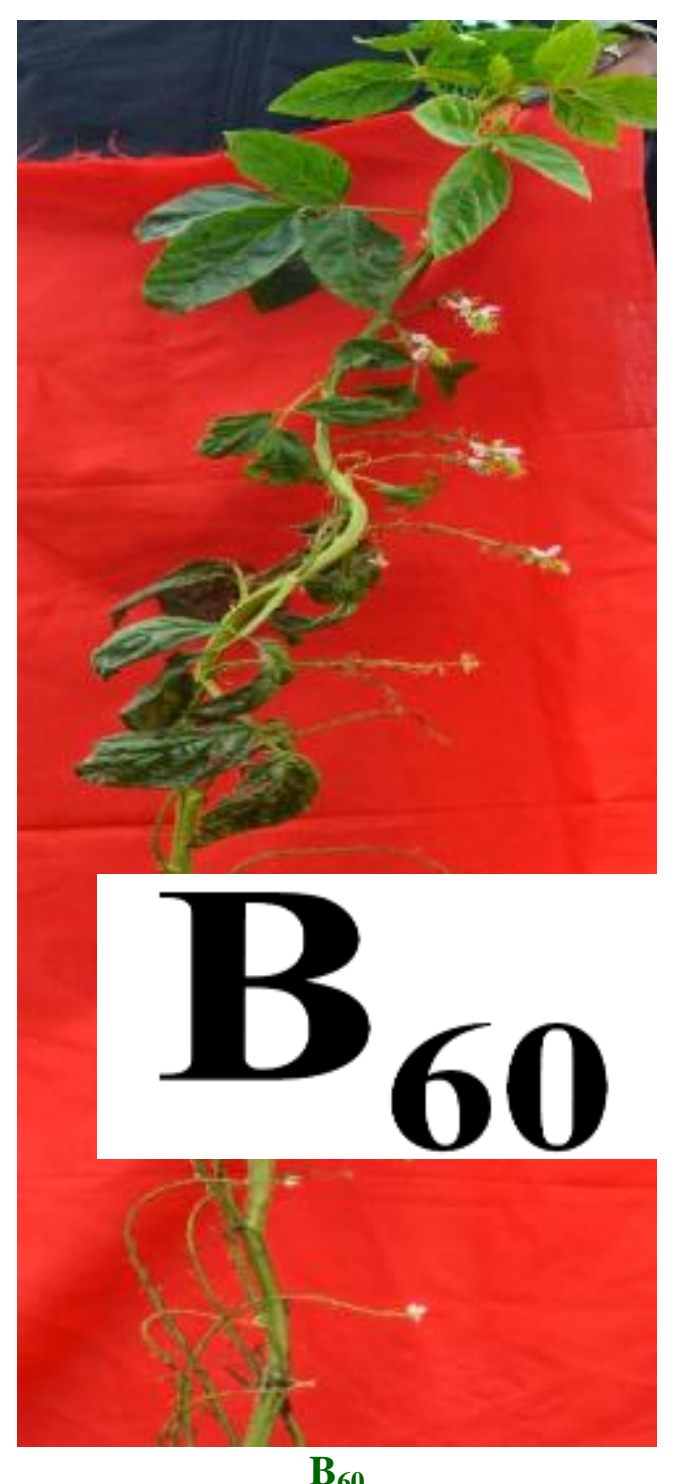

$\mathbf{B}_{60}$
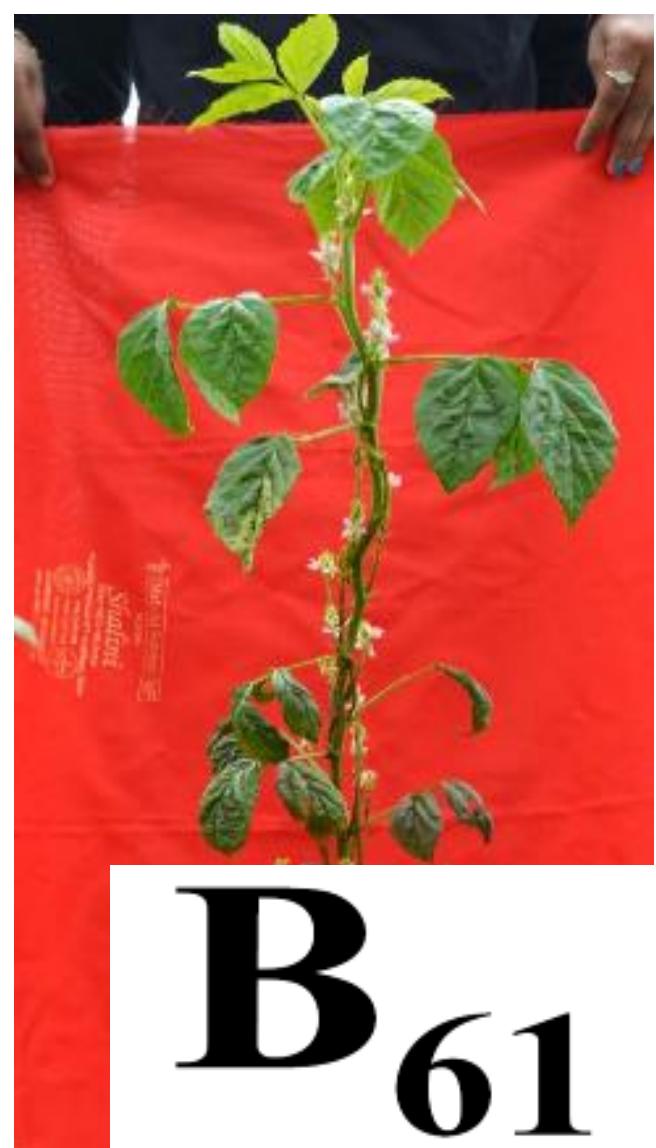

(f

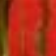

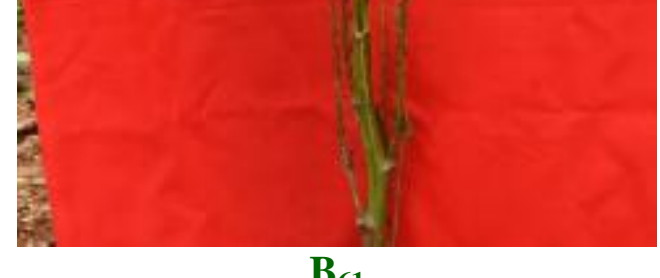

$\mathbf{B}_{61}$ 


\section{Int.J.Curr.Microbiol.App.Sci (2018) 7(11): 1288-1298}

Plate.3 Sterile types observed in $\mathrm{C}$-series of $\mathrm{M}_{4}$ mutants of cluster bean

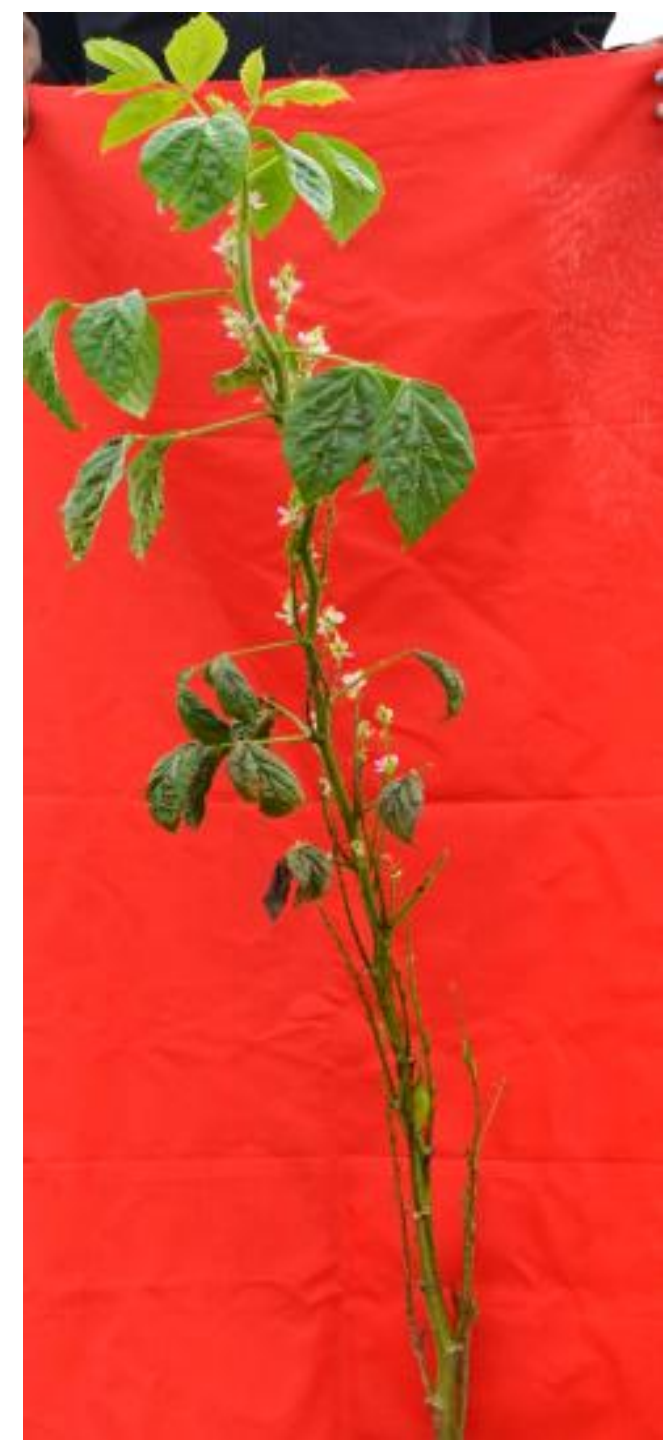

$\mathrm{C}_{93}$

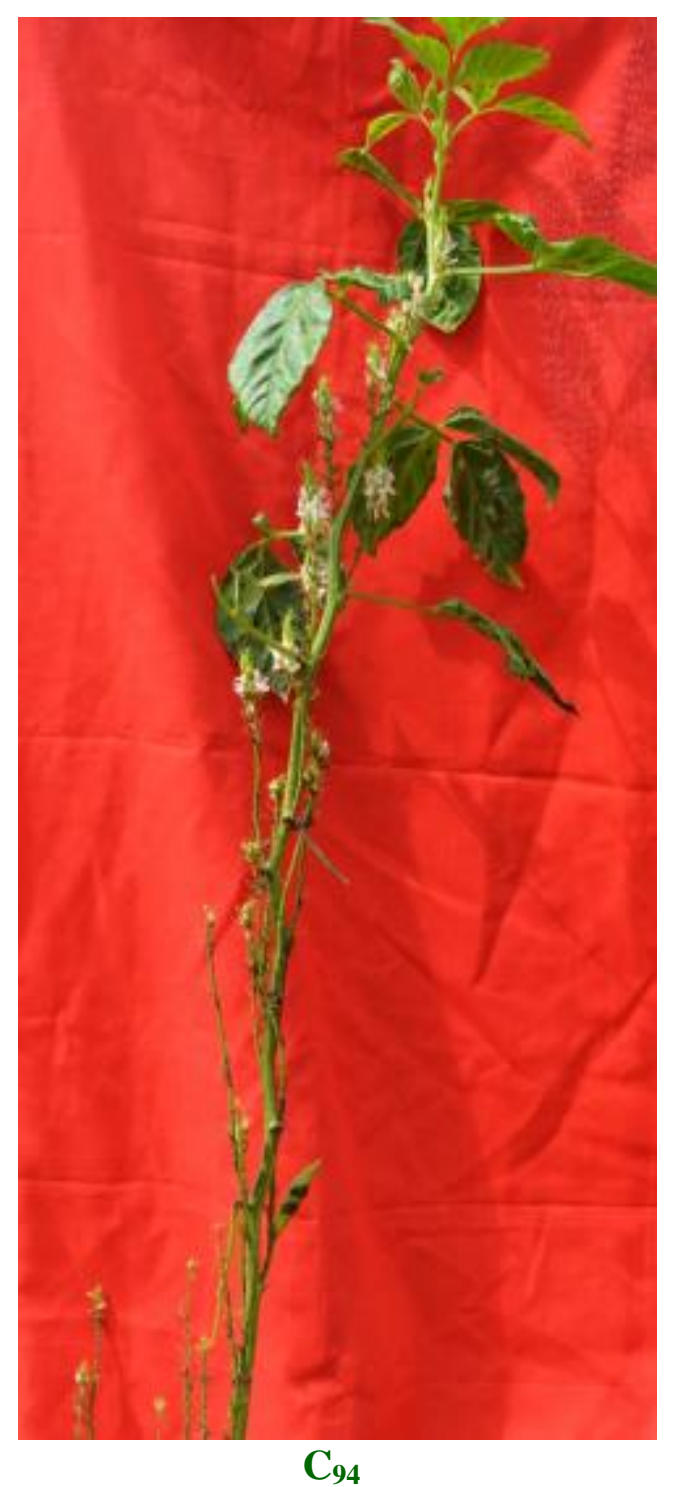

$\mathrm{C}_{94}$

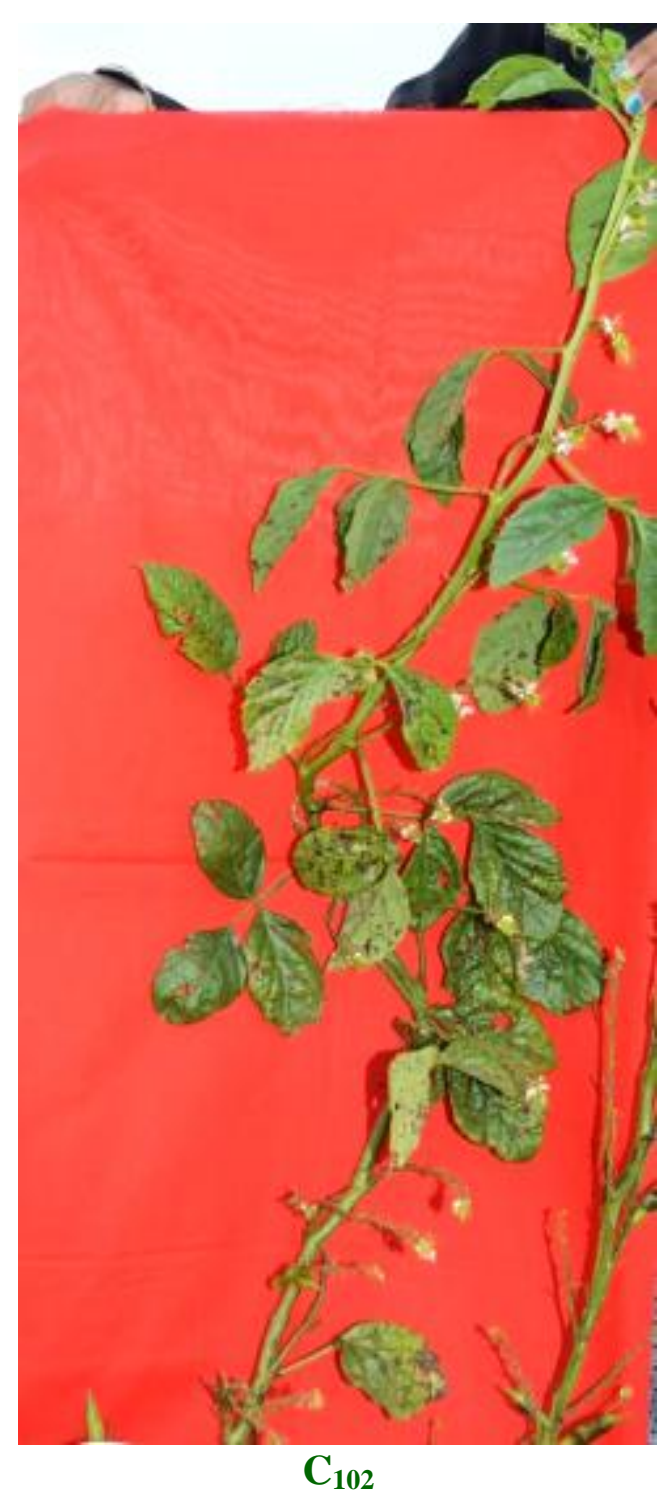

$\mathbf{C}_{102}$ 


\section{Int.J.Curr.Microbiol.App.Sci (2018) 7(11): 1288-1298}

Plate.4 Identified Sterile types observed in D series of $\mathrm{M}_{4}$ mutants of cluster bean
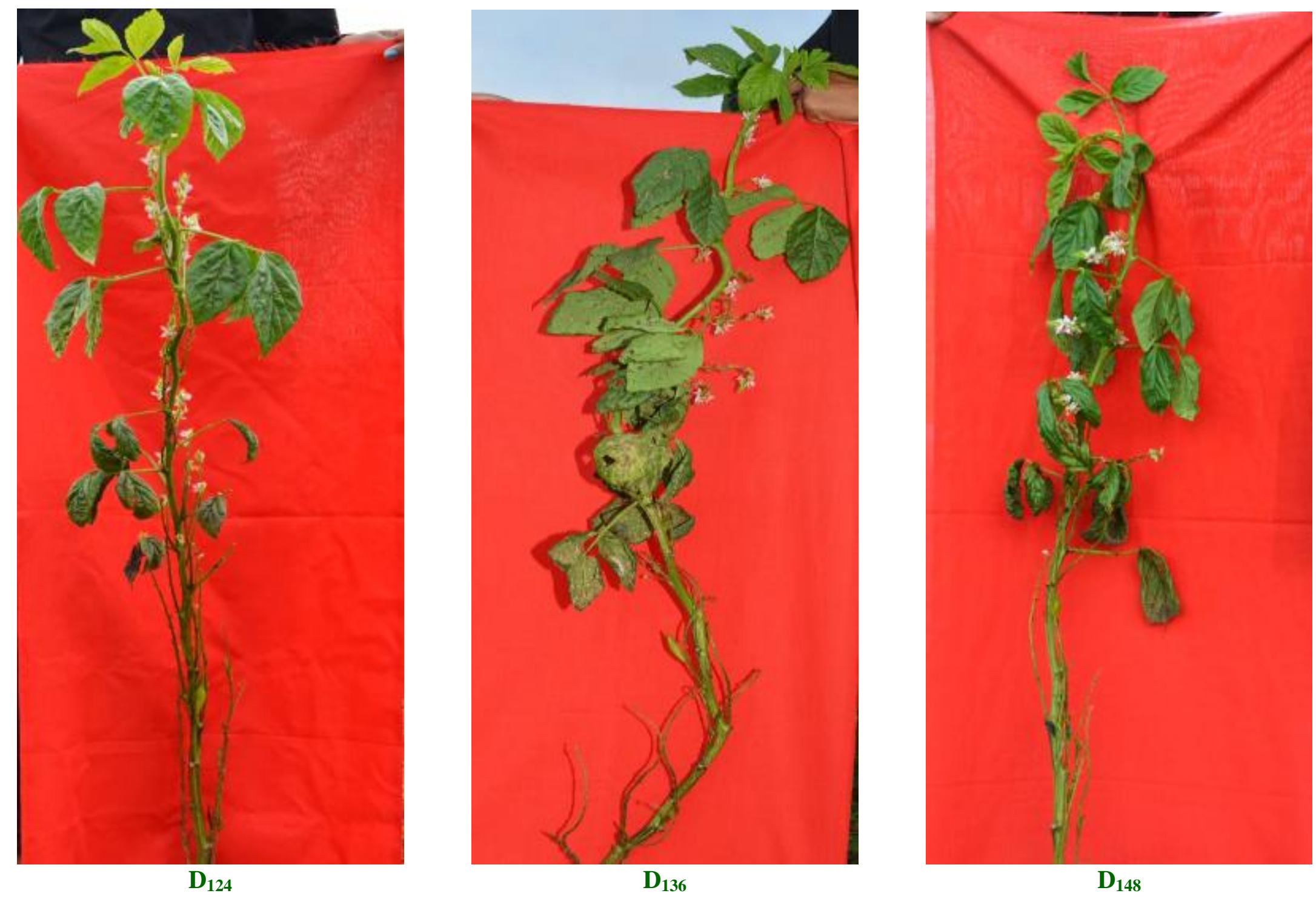
Plate.5 Slide view of Acetocarmine smear test for pollen fertility study in cluster bean

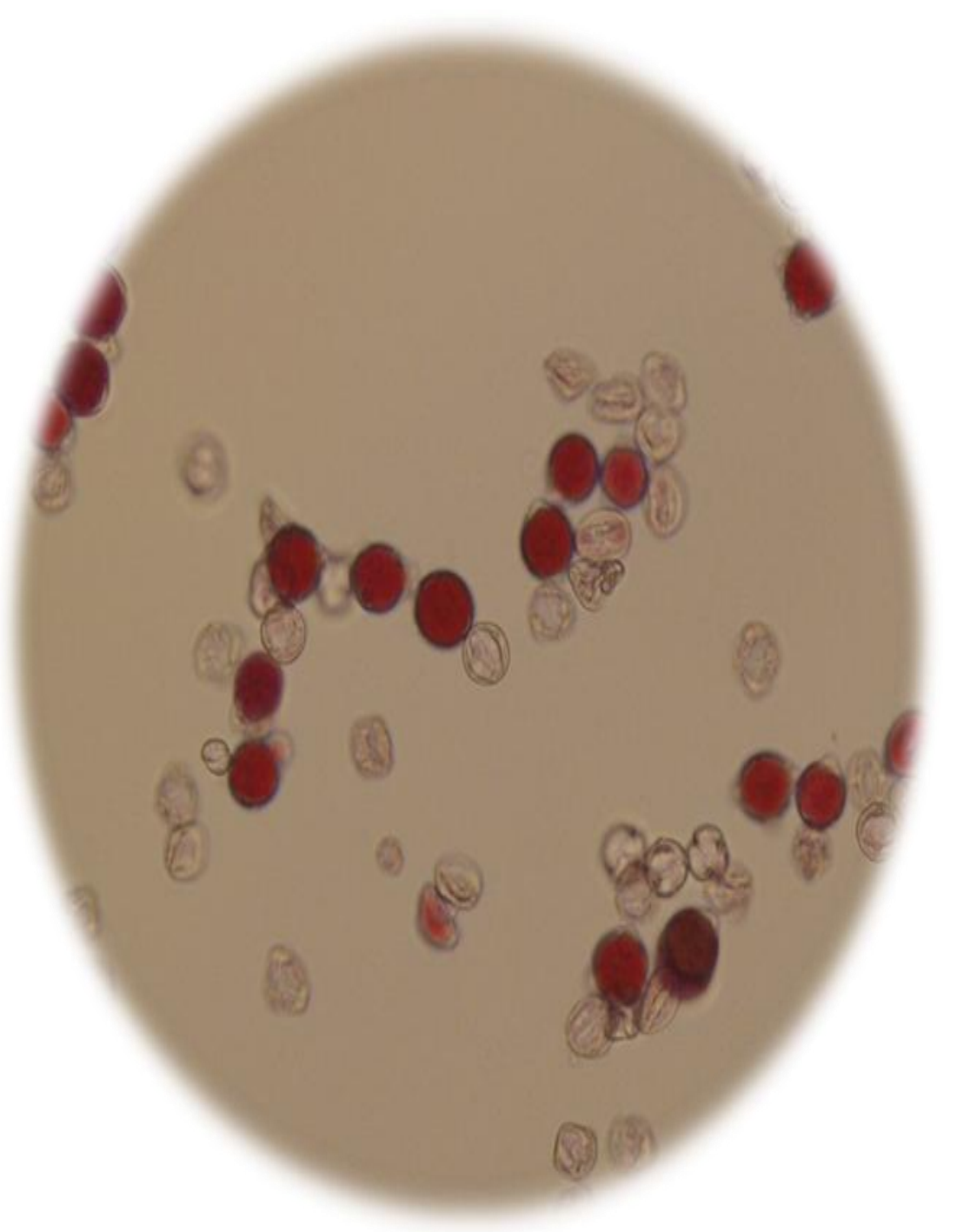

Fertile

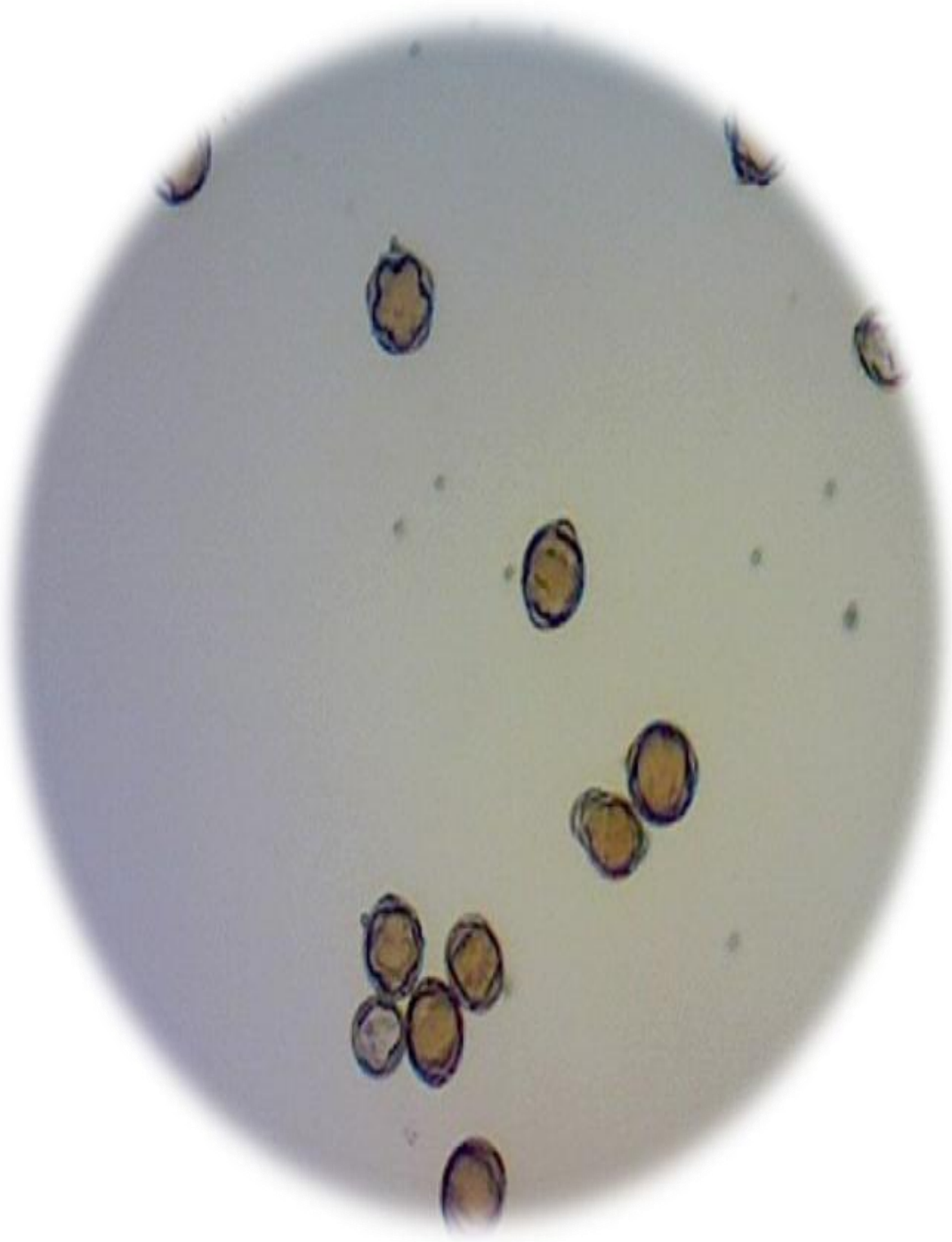

Sterile 
Appendix: Details of mutants used in the study

\begin{tabular}{|c|c|c|c|c|c|c|c|c|}
\hline SI. No. & $\begin{array}{l}\text { Reference } \\
\text { code }\end{array}$ & Mutant name & $\begin{array}{l}\text { Sl. } \\
\text { No. }\end{array}$ & $\begin{array}{l}\text { Referenc } \\
\text { e code }\end{array}$ & Mutant name & $\begin{array}{l}\text { Sl. } \\
\text { No. }\end{array}$ & $\begin{array}{l}\text { Referenc } \\
\text { e code }\end{array}$ & Mutant name \\
\hline 1. & $\mathrm{~A}_{1}$ & $80-17-1$ & 2. & $\mathrm{~B}_{63}$ & 100-ST80-17 & 3. & $\mathrm{D}_{125}$ & $100-16-6$ \\
\hline 4. & $\mathrm{~A}_{2}$ & $80-3-2$ & 5. & $\mathrm{~B}_{64}$ & $100-10-17$ & 6. & $\mathrm{D}_{126}$ & $100-5-12$ \\
\hline 7. & $\mathrm{~A}_{3}$ & $80-24-3$ & 8. & $\mathrm{~B}_{65}$ & $100-5-7$ & 9. & $\mathrm{D}_{127}$ & $100-5-15$ \\
\hline 10. & $\mathrm{~A}_{4}$ & $80-20-3$ & 11. & $\mathrm{~B}_{66}$ & $100-10-4$ & 12. & $\mathrm{D}_{128}$ & $100-5-8$ \\
\hline 13. & $\mathrm{~A}_{5} \mathbf{S}$ & $80-28-3$ & 14. & $\mathrm{~B}_{67}$ & $100-5-14$ & 15. & $\mathrm{D}_{129}$ & $100-5-10$ \\
\hline 16. & $\mathrm{~A}_{6}$ & $80-6-6$ & 17. & $\mathrm{~B}_{68}$ & $100-1-2$ & 18. & $\mathrm{D}_{130}$ & $100-22-7$ \\
\hline 19. & $\mathrm{~A}_{7}$ & $80-9-5$ & 20. & $\mathrm{~B}_{69}$ & 100-ST80-23 & 21. & $\mathrm{D}_{131}$ & $100-25-2$ \\
\hline 22. & $\mathrm{~A}_{8}$ & $80-6-5$ & 23. & $\mathrm{~B}_{70}$ & $100-1-10$ & 24. & $\mathrm{D}_{132}$ & $100-10-3$ \\
\hline 25. & $\mathrm{~A}_{9}$ & $80-3-4$ & 26. & $\mathrm{~B}_{71}$ & $100-1-5$ & 27. & $\mathrm{D}_{133}$ & $100-25-3$ \\
\hline 28. & $\mathrm{~A}_{10}$ & $80-17-5$ & 29. & $\mathrm{~B}_{72}$ & $100-22-1$ & 30. & $\mathrm{D}_{134}$ & $100-2-3$ \\
\hline 31. & $\mathrm{~A}_{11}$ & $80-19-2$ & 32. & $\mathrm{~B}_{73}$ & $100-21-2$ & 33. & $\mathrm{D}_{135}$ & $100-16-5$ \\
\hline 34. & $\mathrm{~A}_{12}$ & $80-17-2$ & 35. & $\mathrm{~B}_{74}$ & $100-2-4$ & 36. & $\mathrm{D}_{136} \mathrm{~S}$ & $100-26-6$ \\
\hline 37. & $\mathrm{~A}_{13}$ & $80-18-17$ & 38. & $\mathrm{~B}_{75}$ & 100-ST80-27 & 39. & $\mathrm{D}_{137}$ & $100-26-1$ \\
\hline 40. & $\mathrm{~A}_{14}$ & $80-27-1$ & 41. & $\mathrm{~B}_{76}$ & 100-ST80-3 & 42. & $\mathrm{D}_{138}$ & $100-5-9$ \\
\hline 43. & $\mathrm{~A}_{15}$ & $80-17-3$ & 44. & $B_{77}$ & $100-22-3$ & 45. & $\mathrm{D}_{139}$ & $100-25-5$ \\
\hline 46. & $\mathrm{~A}_{16} \mathbf{S}$ & $80-18-3$ & 47. & $\mathrm{~B}_{78}$ & $100-5-1$ & 48. & $\mathrm{D}_{140}$ & $100-5-2$ \\
\hline 49. & $\mathrm{~A}_{17}$ & $80-9-2$ & 50. & $\mathrm{~B}_{79}$ & $100-75-7$ & 51. & $\mathrm{D}_{141} \mathrm{~S}$ & $100-26-4$ \\
\hline 52. & $\mathrm{~A}_{18}$ & $80-18-7$ & 53. & $\mathrm{C}_{80}$ & $100-5-13$ & 54. & $\mathrm{D}_{142}$ & $100-10-6$ \\
\hline 55. & $\mathrm{~A}_{19}$ & $80-3-1$ & 56. & $\mathrm{C}_{81}$ & $100-2-5$ & 57. & $\mathrm{D}_{143}$ & $100-21-1$ \\
\hline 58. & $\mathrm{~A}_{20}$ & $80-18-2$ & 59. & $\mathrm{C}_{82}$ & $100-2-7$ & 60. & $\mathrm{D}_{144}$ & $100-22-8$ \\
\hline 61. & $\mathrm{~A}_{21}$ & $80-3-3$ & 62. & $\mathrm{C}_{83}$ & $100-21-5$ & 63. & $\mathrm{D}_{145}$ & $100-10-1$ \\
\hline 64. & $\mathrm{~A}_{22}$ & $80-6-2$ & 65. & $\mathrm{C}_{84}$ & $100-22-6$ & 66. & $\mathrm{D}_{146}$ & $100-1-8$ \\
\hline 67. & $\mathrm{~A}_{23} \mathrm{~S}$ & $80-9-4$ & 68. & $\mathrm{C}_{85}$ & $100-2-6$ & 69. & $\mathrm{D}_{147}$ & $100-25-9$ \\
\hline 70. & $\mathrm{~A}_{24}$ & $80-28-7$ & 71. & $\mathrm{C}_{86}$ & $100-10-8$ & 72. & $\mathrm{D}_{148}$ & $100-2-1$ \\
\hline 73. & $\mathrm{~A}_{25}$ & $80-28-6$ & 74. & $\mathrm{C}_{87}$ & $100-26-5$ & 75. & $\mathrm{D}_{149}$ & $100-1-9$ \\
\hline 76. & $\mathrm{~A}_{26}$ & $80-23-3$ & 77. & $\mathrm{C}_{88}$ & $100-1-4$ & 78. & $\mathrm{D}_{150}$ & $100-5-3$ \\
\hline 79. & $\mathrm{~A}_{27}$ & 80-19-1 & 80. & $\mathrm{C}_{89}$ & $100-16-7$ & 81. & $\mathrm{D}_{151}$ & $100-5-16$ \\
\hline 82. & $\mathrm{~A}_{28}$ & $80-20-6$ & 83. & $\mathrm{C}_{90}$ & $100-26-8$ & 84. & $\mathrm{D}_{152}$ & $100-25-1$ \\
\hline 85. & $\mathrm{~A}_{29}$ & $80-28-1$ & 86. & $\mathrm{C}_{91}$ & $100-16-3$ & 87. & $\mathrm{D}_{153}$ & $100-5-17$ \\
\hline 88. & $\mathrm{~A}_{30}$ & $80-24-5$ & 89. & $\mathrm{C}_{92}$ & $80-24-1$ & 90. & $\mathrm{D}_{154}$ & $100-10-2$ \\
\hline 91. & $\mathrm{~A}_{31}$ & $80-28-2$ & 92. & $\mathrm{C}_{93} \mathrm{~S}$ & $80-9-3$ & 93. & $\mathrm{D}_{155}$ & $100-2-2$ \\
\hline 94. & $\mathrm{~A}_{32}$ & $80-20-4$ & 95. & $\mathrm{C}_{94} \mathrm{~S}$ & $80-6-4$ & 96. & $\mathrm{D}_{156}$ & 100-ST80-22 \\
\hline 97. & $\mathrm{~A}_{33} \mathbf{N P}$ & $80-27-5$ & 98. & $\mathrm{C}_{95} \mathbf{N P}$ & $80-18-5$ & 99. & $\mathrm{D}_{157}$ & 100-ST80-1 \\
\hline 100. & $\mathrm{~A}_{34}$ & $80-20-2$ & 101. & $\mathrm{C}_{96}$ & $80-6-3$ & 102. & $D_{158}$ & 100-ST80-4 \\
\hline 103. & $\mathrm{~A}_{35}$ & $80-20-1$ & 104. & $\mathrm{C}_{97}$ & $80-17-4$ & 105. & $\mathrm{D}_{159} \mathbf{N P}$ & $100-1-7$ \\
\hline 106. & $\mathrm{~A}_{36}$ & $80-27-2$ & 107. & $\mathrm{C}_{98}$ & $80-9-1$ & 108. & $D_{160}$ & $100-25-9$ \\
\hline 109. & $\mathrm{~A}_{37}$ & $80-23-2$ & 110. & $\mathrm{C}_{99}$ & $80-3-5$ & 111. & $\mathrm{D}_{161}$ & $100-10-5$ \\
\hline 112. & $\mathrm{~A}_{38}$ & $80-27-3$ & 113. & $\mathrm{C}_{100}$ & 80-P58-7 & 114. & $\mathrm{D}_{162}$ & $100-25-4$ \\
\hline 115. & $\mathrm{~A}_{39}$ & $80-27-4$ & 116. & $\mathrm{C}_{101}$ & $80-P 58-9$ & 117. & $\mathrm{D}_{163}$ & $100-1-1$ \\
\hline 118. & $\mathrm{~B}_{40}$ & $100-21-3$ & 119. & $\mathrm{C}_{102} \mathrm{~S}$ & 80-P58-8 & 120. & $\mathrm{D}_{164}$ & $100-16-1$ \\
\hline 121. & $\mathrm{~B}_{41}$ & 100-ST80-14 & 122. & $\mathrm{C}_{103}$ & 100-P3-80-1 & 123. & $\mathrm{D}_{165}$ & $100-16-2$ \\
\hline 124. & $\mathrm{~B}_{42}$ & $100-21-4$ & 125. & $\mathrm{C}_{104}$ & 100-P3-80-4 & 126. & $E_{166}$ & 100-MS2-5 \\
\hline 127. & $\mathrm{~B}_{43}$ & 100-ST80-8 & 128. & $\mathrm{C}_{105}$ & $100-P 3-80-2$ & 129. & $E_{167}$ & $100-M S 2-3$ \\
\hline 130. & $\mathrm{~B}_{44}$ & $100-$ ST80-28 & 131. & $\mathrm{C}_{106}$ & $100-\mathrm{P} 3-80-3$ & 132. & $\mathrm{E}_{168}$ & $100-M S 2-1$ \\
\hline 133. & $\mathrm{~B}_{45}$ & 100-ST80-12 & 134. & $\mathrm{C}_{107}$ & 100-A80-4 & 135. & $E_{169}$ & 100-MS2-2 \\
\hline 136. & $\mathrm{~B}_{46}$ & 100-ST80-20 & 137. & $\mathrm{C}_{108}$ & $100-A 80-2$ & 138. & $E_{170}$ & 100-MS2-4 \\
\hline 139. & $\mathrm{~B}_{47}$ & 100-ST80-16 & 140. & $\mathrm{C}_{109} \mathrm{NP}$ & $100-A 80-1$ & 141. & $\mathrm{E}_{171}$ & $100-M S 2-6$ \\
\hline
\end{tabular}




\begin{tabular}{|c|c|c|c|c|c|c|c|c|}
\hline 142. & $\mathrm{~B}_{48}$ & 100-ST80-24 & 143. & $\mathrm{C}_{110}$ & $100-A 80-5$ & 144. & $E_{172}$ & 80-P58-11 \\
\hline 145. & $\mathrm{~B}_{49}$ & 100-ST80-7 & 146. & $\mathrm{C}_{111}$ & $100-A 80-3$ & 147. & $E_{173}$ & 80-P58-3 \\
\hline 148. & $\mathrm{~B}_{50}$ & 100-ST80-13 & 149. & $\mathrm{C}_{112}$ & 100-PNB-1 & 150. & $E_{174}$ & 80-P58-4 \\
\hline 151. & $\mathrm{~B}_{51}$ & $100-25-8$ & 152. & $\mathrm{C}_{113}$ & 100-PNB-2 & 153. & $E_{175}$ & $80-P 58-10$ \\
\hline 154. & $\mathrm{~B}_{52} \mathrm{~S}$ & $100-5-19$ & 155. & $\mathrm{C}_{114}$ & 100-PNB-3 & 156. & $E_{176}$ & 80-P58-1 \\
\hline 157. & $\mathrm{~B}_{53}$ & 100-ST80-21 & 158. & $\mathrm{C}_{115}$ & 100-PNB-4 & 159. & $E_{177}$ & $80-P 58-5$ \\
\hline 160. & $\mathrm{~B}_{54}$ & 100-ST80-15 & 161. & $\mathrm{C}_{116}$ & P58-7 & 162. & $E_{178}$ & 80-P58-2 \\
\hline 163. & $\mathrm{~B}_{55}$ & 100-ST80-10 & 164. & $\mathrm{C}_{117}$ & $100-S T 80-5$ & 165. & $E_{179}$ & 80-P58-6 \\
\hline 166. & $\mathrm{~B}_{56}$ & $100-22-2$ & 167. & $\mathrm{C}_{118}$ & 100-ST80-9 & 168. & $\mathrm{E}_{180}$ & 80-MN2-2 \\
\hline 169. & $\mathrm{~B}_{57}$ & 100-ST80-19 & 170. & $\mathrm{C}_{119}$ & 100-ST80-3 & 171. & $\mathrm{E}_{181}$ & 80-MN2-6 \\
\hline 172. & $\mathrm{~B}_{58}$ & $100-10-11$ & 173. & $\mathrm{C}_{120} \mathrm{NP}$ & 100-ST80-2 & 174. & $\mathrm{E}_{182}$ & 80-MN2-7 \\
\hline 175. & $\mathrm{~B}_{59}$ & 100-ST80-25 & 176. & $\mathrm{C}_{121}$ & 80-MN3-6 & 177. & $\mathrm{E}_{183}$ & 80-MN2-1 \\
\hline 178. & $\mathrm{~B}_{60} \mathrm{~S}$ & 100-ST80-18 & 179. & $\mathrm{C}_{122}$ & 80-MN3-3 & 180. & $\mathrm{E}_{184}$ & 80-MN2-4 \\
\hline 181. & $\mathrm{~B}_{61} \mathrm{~S}$ & 100-ST80-6 & 182. & $D_{123}$ & $100-1-6$ & 183. & $\mathrm{E}_{185}$ & $80-\mathrm{MN} 2-5$ \\
\hline 184. & $\mathrm{~B}_{62}$ & 100-ST80-11 & 185. & $\mathrm{D}_{124} \mathrm{~S}$ & $100-26-7$ & 186. & $\mathrm{E}_{186}$ & 80-MN3-5 \\
\hline 187. & $E_{188}$ & 80-MN3-2 & 188. & $\mathrm{E}_{189}$ & 80-MN3-1 & 189. & $E_{187}$ & 80-MN3-7 \\
\hline 190. & $E_{190}$ & 80-MN3-3 & 191. & & & 192. & & \\
\hline
\end{tabular}

The completely sterile plants were characterized by the determinate growth habit with highest plant height and elongated peduncles having one or two seeded pods or no pods along the entire plant height and gamma rays revealed the maximum sterility in cluster bean was noticed by Shinde et al., (2010). Highest plant height observed in case of sterile mutants $i$. e., $96.60 \mathrm{~cm}$. ranges for different traits is plant height (61.40 to 96.60 $\mathrm{cm}$ ), number of branches (14.80 to 23.00), number of days to 50 per cent flowering (26.00 to 36.00) and the values for other quantitative traits is 0.00 because no pods are developed in the sterile mutants (Table 1). The sterility was confirmed and the pollen fertility percentage was recorded and calculated by doing the Acetocarmine smear test. The results revealed that the stained pollens are considered as fertile and nonstained pollens are sterile because the pollens are non-viable in case of sterile plants (Plate 5) and the fertile mutants are observed with $82.02 \%\left(\mathrm{D}_{160}\right)$ to $99.72 \%\left(\mathrm{D}_{163}\right)$ per cent pollen fertility while, the pollen fertility was $5.06 \%\left(\mathrm{~B}_{60}\right)$ to $10.04 \%\left(\mathrm{D}_{141}\right)$ in case of sterile plants.

The following are the sterile mutants observed in the present study $\mathrm{A}_{5}, \mathrm{~A}_{16}, \mathrm{~A}_{23}$,
$\mathrm{B}_{52}, \mathrm{~B}_{60}, \mathrm{~B}_{61}, \mathrm{C}_{93}, \mathrm{C}_{94}, \mathrm{C}_{102}, \mathrm{D}_{124}, \mathrm{D}_{136}$ and $\mathrm{D}_{141}$. The completely sterile mutant plants morphologically differ from fertile mutants i.e., characterized by the determinate habit with elongated peduncles having only flowering is observed and no pod setting and development with the more vegetative growth and very less amount reproductive growth is noticed in these mutants with one or two seeded pods Highest plant height observed in case of sterile mutants $i$. e., $96.60 \mathrm{~cm}$. ranges for different traits is plant height (61.40 to $96.60 \mathrm{~cm}$ ), number of branches (14.80 to 23.00 ), number of days to 50 per cent flowering (26.00 to 36.00) and the values for other quantitative traits is 0.00 because no pods are developed in the sterile mutants. The sterility was confirmed and the pollen fertility percentage was recorded and calculated by doing the acetocarmine smear test. And also observed with 82.02 per cent $\left(\mathrm{D}_{160}\right)$ to 99.72 per cent $\left(\mathrm{D}_{163}\right)$ per cent pollen fertility while, the pollen fertility was 5.06 per cent $\left(\mathrm{B}_{60}\right)$ to 10.04 per cent $\left(\mathrm{D}_{141}\right)$.

\section{Acknowledgements}

Authors are thankful to Dean Dr. G. Janardhan and Farm superintendent Dr. Yathindra at College of Horticulture, Mysuru, 
Karnataka, Dr. Mukesh L. Chavan, Profesor and Head Department of Biotchnology and Crop Improvement, Dr. V. Nachegowada, Director of Research UHS Bagalkot, Dr. N. Basavaraja Dean (Post Graduate Studies) at UHS Bagalkot for their support during the time of this investigation, Authors are also thankful to all the III B.Sc.(Horti) students of College of Horticulture for extending timely help.

\section{References}

Anila, G. and Balakrishnan, R., 1990, Variability studies in cluster bean (Cyamopsis tetragonoloba (L.) Taub.). South Indian Hort., 38(6): 311-314.

Anonymous, 2016, Package of practice, 9596.

Dabas, B. S., Mital, S. P. and Arunachalam, V., 1982. An evaluation of germplasm accessions in guar. Indian J. Genet, 42: 56-59.

Girish, M. H., Gasti, V. D., Thammaiah, N., Kerutagi, M. G, Mulge, R, Shantappa, T. and Mastiholi, A. B., 2012, Genetic divergence studies in cluster bean genotypes [Cyamopsis tetragonoloba
(L.) Taub.]. Karnataka J. Agric. Sci., 25(2): 245-247.

Hanchinamani, N. C., 2004, Studies on genetic variability and genetic divergence in cluster bean (Cyamopsis tetragonoloba (L.) Taub.). M. Sc. (Hort.) Thesis, Univ. Agric. Sci., Dharwad.

Saini, D. D., Singh, N. P., Chaudhary, S. P. S., Chaudhary, O. P and Khedar, O. P., 2010, Genetic variability and association of component characters for seed yield in cluster bean [Cyamopsis tetragonoloba (L.) Taub.]. J. Arid Legumes, 7(1): 47-51.

Sanghi, A. K., Bhatnagar, M. P. and Sharma, S. K., 1964, Genotypic and phenotypic variability in yield and other quantitative characters in guar. Indian J. Genet., 24(2): 164-167.

Shinde, M. S. and More, A. D., 2010, Study of pollen sterility in cluster bean [Cyamopsis tetragonoloba (L.) Taub.] through mutagenesis. Asian J. Exp. Biol. Sci. Spl., 31-34.

Tawar, M. L., Mishra, A. K., Rao, S. K. and Sharma, S. M., 1988, Genetic divergence in mung bean. Legume Res., 11(3): 109-113.

\section{How to cite this article:}

Meghana, N.M., R.K. Ramachandra, B. Fakrudin, H.M. Pallavi, Vishnuvardhana, M. Anjanappa and Harish, A. 2018. Spikelet Sterility Studies on Induced Mutant Populations of Cluster Bean [Cyamopsis tetragonoloba (L.) Taub.]. Int.J.Curr.Microbiol.App.Sci. 7(11): 12881298. doi: https://doi.org/10.20546/ijcmas.2018.711.150 